\title{
The Therapeutic Effects of Exosomes Derived from Human Umbilical Cord Mesenchymal Stem Cells on Scleroderma
}

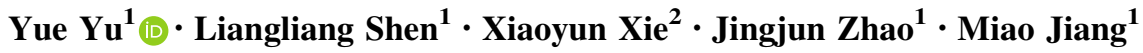

Received: 28 August 2021/Revised: 27 September 2021/Accepted: 2 October 2021/Published online: 16 November 2021

(C) The Author(s) 2021

\begin{abstract}
BACKGROUND: Scleroderma is a multisystem disease in which tissue fibrosis is caused by inflammation and vascular damage. The mortality of scleroderma has remained high due to a lack of effective treatments. However, exosomes derived from human umbilical cord mesenchymal stem cells (HUMSCs)-Ex have been regarded as potential treatments for various autoimmune diseases, and may also act as candidates for treating scleroderma.

METHODS: Mice with scleroderma received a single $50 \mu \mathrm{g}$ HUMSCs-Ex. HUMSCs-Ex was characterized using transmission electron microscopy, nanoparticle tracking analysis and nanoflow cytometry. The therapeutic efficacy was assessed using histopathology, immunohistochemistry, immunofluorescence, quantitative real-time polymerase chain reaction, enzyme-linked immunosorbent assay and western blot.

RESULTS: HUMSCs-Ex ameliorated the deposition of extracellular matrix and suppressed the epithelial-mesenchymal transition process, and the effects lasted at least three weeks. In addition, HUMSCs-Ex promoted M1 macrophage polarization and inhibited M2 macrophage polarization, leading to the restoration of the balance of M1/M2 macrophages. CONCLUSION: We investigated the potential antifibrotic and anti-inflammatory effects of HUMSCs-Ex in a bleomycininduced mouse model of scleroderma. So HUMSCs-Ex could be considered as a candidate therapy for scleroderma.
\end{abstract}

Keywords Scleroderma $\cdot$ Human umbilical cord mesenchymal stem cell $\cdot$ Exosome $\cdot$ Macrophage $\cdot$ Bleomycin

\section{Introduction}

Scleroderma is an autoimmune tissue disease characterized by vascular dysfunction, inflammatory disorder and extensive fibrosis [1]. Although the prevalence $(7 \sim 489 / 1$

Yue $\mathrm{Yu}$ and Liangliang Shen have contributed equally to this work.

Jingjun Zhao

zhaojingjun2015@aliyun.com

$\triangle$ Miao Jiang

jiang_miao2014@126.com

1 Department of Dermatology, Tongji Hospital, School of Medicine, Tongji University, Shanghai 200065, China

2 Department of Interventional and Vascular Surgery, Shanghai Tenth People's Hospital, School of Medicine, Tongji University, Shanghai 200072, China million $)$ and the incidence of $(0.6 \sim 122 / 1$ million $)$ scleroderma are low [2], the death rate remains high [3]. The 10 -years survival rate is only $66 \%$, and the quality of life of survivors is significantly affected [4]. The main reason for the poor outcomes of scleroderma is that fibrosis destroys the normal structures of the skin, lung, kidney and skeletal system, resulting in severe complications such as pulmonary hypertension, acute kidney injury and acro-osteolysis [5].

Macrophages are involved in the process of scleroderma [6]. After infiltrating the tissue, monocytes can differentiate into macrophages depending on the microenvironment. Differentiated macrophages can be divided into classical activated inflammatory macrophages (M1) and tissue-activated fibrotic macrophages (M2). M1 macrophages promote inflammation by releasing TNF- $\alpha$, IL-6, and IL- 12 . 
M2 macrophages stimulate fibrosis by releasing IL-4, IL10 and IL-13. Overall, regulation of the M1/M2 macrophage balance is a strategy for the treatment of scleroderma [7]. Epithelial-mesenchymal transition (EMT) is a process in which stable epithelial cells lose cell-cell adhesion and acquire mesenchymal characteristics, and the main mesenchymal cells in skin are fibroblasts. Activation of fibroblasts is an important process of promoting extracellular matrix (ECM) deposition, which is realized by differentiation into myofibroblasts [8].

Stem cells are a kind of cell with the potential for selfrenewal and multidirectional differentiation, so they have been used in the repair of various tissues and organs. Multiple studies have shown that mesenchymal stem cells (MSCs) mainly rely on paracrine factors to play a therapeutic role, and exosomes are an important component of paracrine factors $[9,10]$. Exosomes are vesicles filled with a variety of proteins, microRNAs, and DNA that perform biological functions on target cells [11]. In addition, HUMSCs-Ex transplantation is a cell-free therapy, that has the characteristics of low immune source and high stability [12]. Studies have shown that exosomes can treat various diseases by regulating EMT [13] or macrophage balance [14], but the mechanism of exosomes in scleroderma remains unclear. In this study, we confirmed that HUMSCs-Ex had an effect on the treatment of skin fibrosis and the regulation of macrophage balance in a mouse model of scleroderma.

\section{Materials and methods}

\subsection{Exosomes purification and characterization}

Human umbilical cord mesenchymal stem cells were obtained from Shanghai Tenth People's Hospital and cultured in DMEM/F12 complete medium (Thermo Fisher Scientific, Shanghai, China) at $5 \% \mathrm{CO}_{2}$ and $37{ }^{\circ} \mathrm{C}$. The cells at passage four were used for experiments. The cell culture supernatant was centrifuged at $2000 \times \mathrm{g}$ for $30 \mathrm{~min}$ to eliminate cell debris. After centrifugation at $10,000 \times \mathrm{g}$ for $45 \mathrm{~min}$, the supernatant was filtered through a $0.22-\mu \mathrm{m}$ filter (Millipore, Burlington, MA, USA). The supernatant was transferred to new tubes and centrifuged at $100,000 \times \mathrm{g}$ for $70 \mathrm{~min}$ in an ultracentrifuge (Hitachi, Tokyo, Japan). After a second ultracentrifugation, exosomes were resuspended in $100 \mu \mathrm{L}$ of cold PBS and taken for subsequent identification. The concentration of HUMSCs-Ex was measured using a BCA protein assay kit (Beyotime, Shanghai, China). Transmission electron microscopy (TEM)(Hitachi), nanoparticle tracking analysis (NTA)(NanoFCM, Xiamen, China) and nanoflow cytometry (nFCM)(NanoFCM) were used to analyze the morphology and surface markers of HUMSCs-Ex.

\subsection{Preparation of a mouse model of scleroderma}

Female Balb/c mice aged 6 to 8 weeks were purchased from Shanghai SLAC Laboratory Animal Center. Female Balb/c mice aged 6 to 8 weeks were purchased from Shanghai SLAC Laboratory Animal Center. We did not explore the effects of the gender of the mice on results, but female mice are commonly used in the bleomycin-induced mouse model of scleroderma. Mice were randomly divided into the control group and the bleomycin-induced group. Mice in the bleomycin-induced group were subcutaneously injected with $100 \mu \mathrm{l}$ bleomycin (Thermo Fisher Scientific) at $1 \mathrm{mg} / \mathrm{mg}$ once a day for 4 weeks. Mice in the control group were injected with $100 \mu \mathrm{L}$ phosphate buffered saline (PBS) for 4 weeks. On day 28, the bleomycin-induced group was randomly divided into the exosome (Ex) group and the new bleomycin-induced (BLM) group. Mice in the Ex group were subcutaneously injected on their backs with $100 \mu \mathrm{L}$ of PBS containing $50 \mu \mathrm{g}$ of HUMSCs-Ex, while the control group and BLM group were injected with 100 $\mu \mathrm{L}$ PBS. Mice were randomly sacrificed, and skin and blood samples were collected at 1, 2, and 3 weeks after HUMSCs-Ex or PBS injection.

\subsection{Histological assessment}

Specimens from skin lesions were fixed overnight with $4 \%$ paraformaldehyde for $24 \mathrm{~h}$ at $4{ }^{\circ} \mathrm{C}$, paraffin-embedded and sectioned at $4 \mu \mathrm{m}$. The sections were stained with hematoxylin \& eosin (H\&E) or Masson's trichrome to assess the dermal thickness and collagen content, respectively. The histologic sections were scanned by pannoramic scan (3DHISTECH, Budapest, Hungary). Then the images were observed and photographed using CaseViewer software (3DHISTECH, Hungary) at $100 \times$ magnification and quantified in Image $\mathbf{J}$ software (National Institutes of Health, Bethesda, MD, USA). Three microscopic fields were selected for each section. Dermal thickness was defined as the distance between the epidermal-dermal junction and the dermal-subcutaneous fat junction. Collagen content was defined as the percentage of collagen deposition area to total area.

\subsection{Immunohistochemical analysis}

The paraffin-embedded skin sections were incubated with $3 \% \mathrm{H}_{2} \mathrm{O}_{2}$ for $10 \mathrm{~min}$ and then $10 \%$ normal goat serum albumin (Solarbio, Beijing, China) for $10 \mathrm{~min}$. The sections were stained with mouse monoclonal antibodies against $\alpha$-SMA (1:200 dilution, BOSTER, Wuhan, China), Vimentin (1:200 dilution, BOSTER), E-cadherin (1:200 dilution, Cell Signaling Technology, Danvers, MA, USA) and TGF- $\beta 1$ (1:200 dilution, BOSTER) overnight at $4{ }^{\circ} \mathrm{C}$. 
The sections were incubated with biotinylated secondary antibodies for $30 \mathrm{~min}$ at room temperature. Diaminobenzidine solution was used as a chromogen to visualize the immunostaining results. The immunohistochemistry sections were scanned by pannoramic scan. Then, the images were observed and photographed using CaseViewer software at $400 \times$ magnification, and quantified in Image-Pro Plus software (Media Cybernetics, Rockville, MD, USA). Five microscopic fields were selected for each section, and the mean intensity optical density (IOD) was used to quantify the protein expression level.

\subsection{Immunofluoresence analysis}

The paraffin-embedded skin sections were blocked with $10 \%$ normal goat serum albumin and incubated with mouse antiiNOS antibody (1:200 dilution, Abcam, Cambridge, MA, USA) or rabbit anti-CD206 antibody (1:200 dilution, Abcam), and rabbit anti-F4/80 antibody (1:200 dilution, Abcam) overnight at $4{ }^{\circ} \mathrm{C}$. The sections were incubated with Alexa Fluor 488 AffiniPure goat anti-rabbit IgG (1:500 dilution, Jackson ImmunoResearch, West Grove, PA, USA) or Alexa Fluor 488-conjugated AffiniPure goat anti-mouse IgG (1:500 dilution, Jackson ImmunoResearch) and Cy3-conjugated AffiniPure goat anti-rabbit IgG (1:500 dilution, Jackson, USA) at for $1 \mathrm{~h}$. Then nuclei were stained with 6-diamidino-2phenylindole. The sections were observed by fluorescence microscopy (Nikon, Shanghai, China) and scanned by pannoramic scan. Then the double positive cells were counted by CaseViewer software at $630 \times$ magnification and five microscopic fields were selected for each section.

\subsection{Cytokine analysis by enzyme-linked immunosorbent assay (ELISA)}

The levels of IL-4, IL-6, IL-10 and IL-12p40 in serum were quantified by ELISA kits (BOSTER) according to the manufacturer's instructions. The absorbance was determined at $450 \mathrm{~nm}$ by an ELISA reader (Rayto, Shenzhen, China) and the actual concentration was then calculated using the standard curve.

\subsection{Quantitative real-time polymerase chain reaction (qPCR)}

Total RNA was extracted from skin samples using TRIzol reagent (Invitrogen, Waltham, MA, USA). Complementary DNAs (cDNAs) were reverse transcribed using the PrimeScript ${ }^{\mathrm{TM}}$ RT reagent Kit (TaKaRa, Japan). qPCR was performed with AceQ Universal SYBR qPCR Master Mix (Vazyme Biotech Co.,Ltd, Nanjing, China) in a BIOER 9600 real-time PCR system. Target gene expression levels were calculated using the $2-\Delta \Delta \mathrm{Ct}$ method, and were normalized to ACTB. The primers used were as follows: coll $\alpha 1,5^{\prime}-$ CTGGCGGTTCAGGTCCAAT- $3^{\prime}$ (forward primer) and $5^{\prime}$ TTCCAGGCAATCCACGAGC- $3^{\prime}$ (reverse primer); col $3 \alpha 1$, 5'-CTGTAACATGGAAACTGGGGAAA- $3^{\prime}$ (forward primer) and $5^{\prime}$-CCATAGCTGAACTGAAAACCACC-3' (reverse primer); ACTB, 5'-GTGACGTTGACATCCGTAAA GA- $3^{\prime}$ (forward primer) and $5^{\prime}$-GCCGGACTCATCGTA CTCC- $3^{\prime}$ (reverse primer).

\subsection{Western blot}

Skin samples were lysed with RIPA buffer (BOSTER) and PMSF (BOSTER) to extract protein. The concentration of protein was determined with a BCA protein assay kit (BOSTER). An equivalent amount of protein was loaded onto 10\% SDS-PAGE gels (BOSTER), electrophoretically treated and then transferred to PVDF membranes (Millipore). The membranes were incubated with $5 \%$ nonfat milk in TBS-T for $2 \mathrm{~h}$ at room temperature, and then incubated with primary antibodies at $4{ }^{\circ} \mathrm{C}$ overnight. The membranes were incubated with secondary antibodies for $2 \mathrm{~h}$ at room temperature, and the protein bands were visualized using ECL reagents. Finally, the bands were photographed using gel image analysis system (Tanon Science \& Technology Co., Shanghai, China) and the intensity of the bands was quantified in Gel-Pro analyzer software (Media Cybernetics). The details of the primary and secondary antibodies are as follows: anti-iNOS (1:1000, Thermo Fisher Scientific), anti-Arg-1 (1:500, BOSTER), and horseradish peroxidase-conjugated AffiniPure goat anti-rabbit $\mathrm{IgG}$ (1:2000, Jackson ImmunoResearch).

\subsection{Statistical analysis}

All statistical analyses were performed using GraphPad Prism software version 8.0.1 (GraphPad Software, San Diego, CA, USA). One-way ANOVA was used to analyze the significant differences when multiple experimental groups were compared. $P$ values $<0.05$ were considered statistically significant. Data are presented as the mean $\pm \mathrm{SD}$.

\section{Results}

\subsection{Characterization of HUMSCs-Ex}

TEM images showed that HUMSCs-Ex presented a globulare shape in Fig. 1 and a saucer-shaped morphology, as shown in Fig. 1B. NTA showed that the mean diameter of HUMSCs-Ex was $140.6 \mathrm{~nm}$ (Fig. 1C), which was consistent with previously reported exosomes. nFCM revealed that the expression levels of CD9, CD63 and CD81 were 

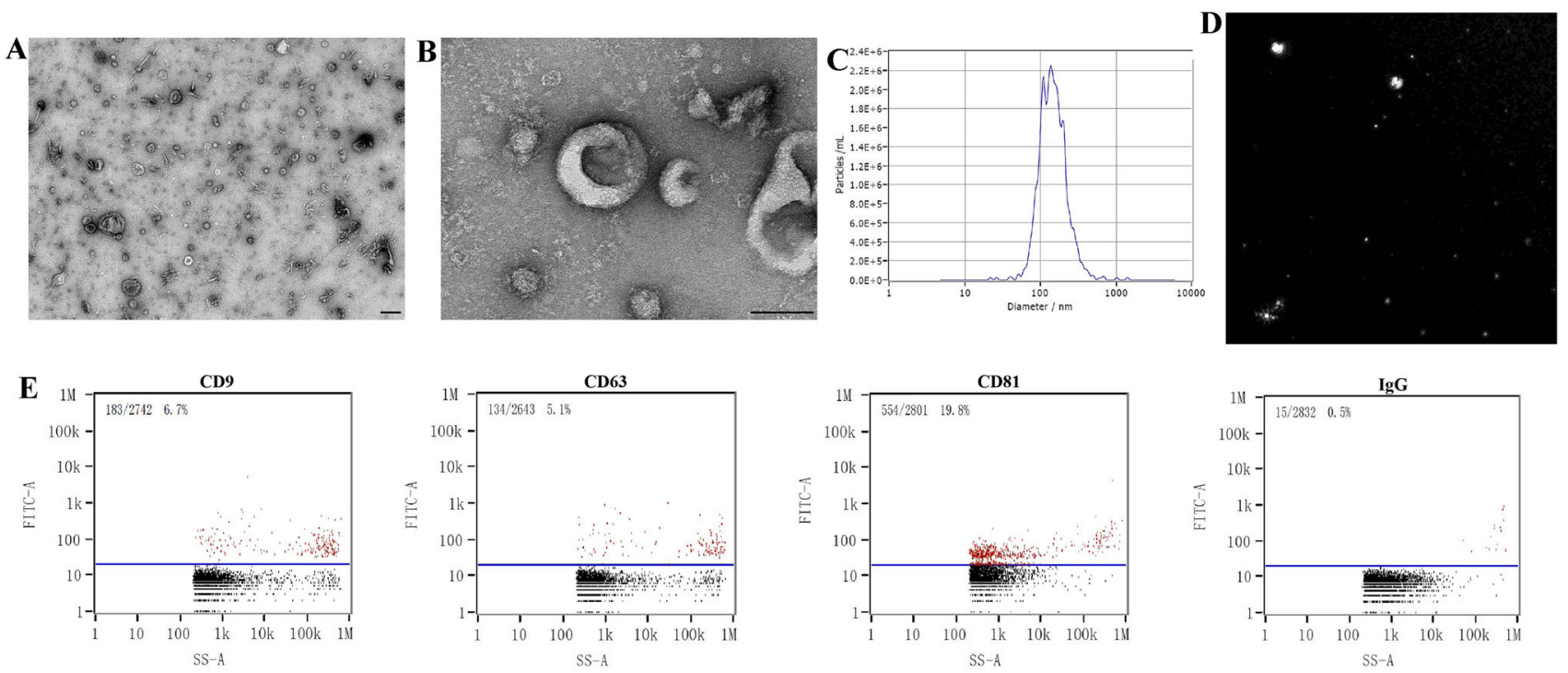

Fig. 1 A, B Identification of HUMSCs-Ex. Representative images of HUMSCs-Ex under TEM. Scale bars $=200 \mathrm{~nm}(\mathbf{A})$ and $100 \mathrm{~nm}(\mathbf{B})$. C NTA of HUMSCs-Ex diameters. D NTA image of HUMSCs-Ex.

obviously higher than that of the IgG isotype control (Fig. 1D).

\subsection{HUMSCs-Ex ameliorated skin fibrosis in a mouse model of scleroderma}

As shown in Fig. 2, the epidermis, dermis and subcutaneous fat layer were clearly differentiated in the control group, and there was no inflammatory cell infiltration. Compared with the control group, the dermal thickness increased significantly and the subcutaneous fat layer partially disappeared in the BLM group. HUMSCs-Ex reduced the dermal thickness (Fig. 2D). As shown in Fig. 2B, collagen fibers were arranged regularly in the control group. Compared with the control group, collagen fibers in the BLM group were thickened and replaced the subcutaneous fat layer to some extent. HUMSCs-Ex significantly reduced the collagen content at 1 week and 2 weeks but not at 3 weeks, which might be caused by HUMSCs-Ex not restoring the subcutaneous fat layer (Fig. 2E). Collagen is a marker of ECM and is used to assess the extent of collagen deposition. As shown in Fig. 2F-G, the relative expression of coll $\alpha 1$ and col3 $\alpha 1$ mRNA in the BLM group were higher than that in the control group. HUMSCs-Ex reduced the transcription level of col1 $\alpha 1$ and col3 $\alpha 1$ mRNA. TGF$\beta 1$ is believed to be a central mediator in skin fibrosis and a biomarker for predicting the progression of scleroderma [19]. Compared with the control group, the IOD score of TGF- $\beta 1$ was elevated in the BLM group (Fig. 2C). HUMSCs-Ex reduced the expression of TGF- $\beta 1$ (Fig. $2 \mathrm{H}$ ). The above changes in dermal thickness, and TGF- $\beta 1$,

E CD9, CD63 and CD81 expression on single HUMSCs-Ex using FITC by nFCM. An IgG isotype control was used to establish the nonspecific binding of antibodies

col1 $\alpha 1$ and col3 $\alpha 1$ mRNA expression levels were similar except for collagen content at 1 week, 2 weeks and 3 weeks.

\subsection{HUMSCs-Ex inhibited skin EMT in a mouse model of scleroderma}

To explore the influence of HUMSCs-Ex on EMT, we performed immunohistochemistry analysis of E-cadherin, a marker of endothelial tissue, and Vimentin, a marker of mesenchymal tissue. We also studied $\alpha$-SMA, a marker of myofibroblasts. The immunohistochemistry results revealed an increased expression of Vimentin and a decreased expression of E-cadherin in the BLM group compared with the control group, and HUMSCs-Ex decreased the IOD scores of Vimentin and increased the IOD scores of E-cadherin. Compared with the control group, a larger number of $\alpha-\mathrm{SMA}^{+}$myofibroblasts was found in the BLM group than in the control group. HUMSCs-Ex reduced the number of $\alpha$-SMA ${ }^{+}$myofibroblasts. The above changes in Vimentin, E-cadherin and $\alpha$ SMA were similar at 1 week, 2 weeks and 3 weeks (Fig. 3).

\subsection{HUMSCs-Ex regulated the balance of M1/M2 macrophages in a mouse model of scleroderma.}

To study the effect of HUMSCs-Ex on the balance of M1/ M2 macrophages, skin and serum samples of mice killed in the first week were tested for related indicators. Compared with the control group, the protein levels of IL- 6 and IL-12 

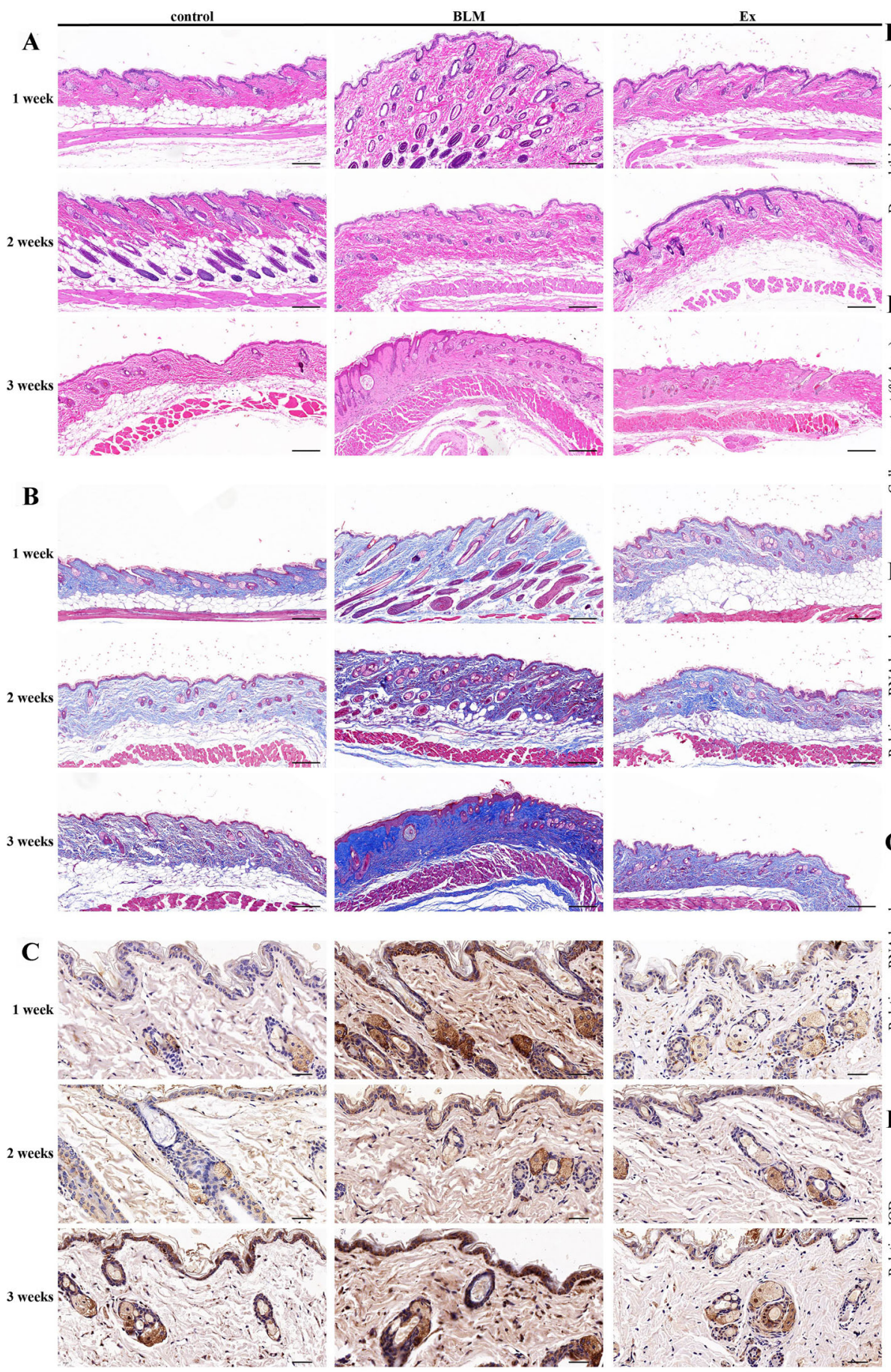

Fig. 2 HUMSCs-Ex alleviated skin fibrosis in a bleomycin-induced mouse model of scleroderma. A, B The respective images are the skin sections stained with H\&E and Masson's trichrome. Scale bars = $200 \mu \mathrm{m}$. C The distribution and expression of TGF-b1 were assessed by immunohistochemical staining. Scale bars $=40 \mu \mathrm{m}$. D-E Dermal

secreted by M1 macrophages were downregulated in the BLM group. In contrast, the protein levels increased in the Ex group, but the changes in IL-6 were not statistically significant ( $p>0.05$ vs. the BLM group) (Fig. 4). The

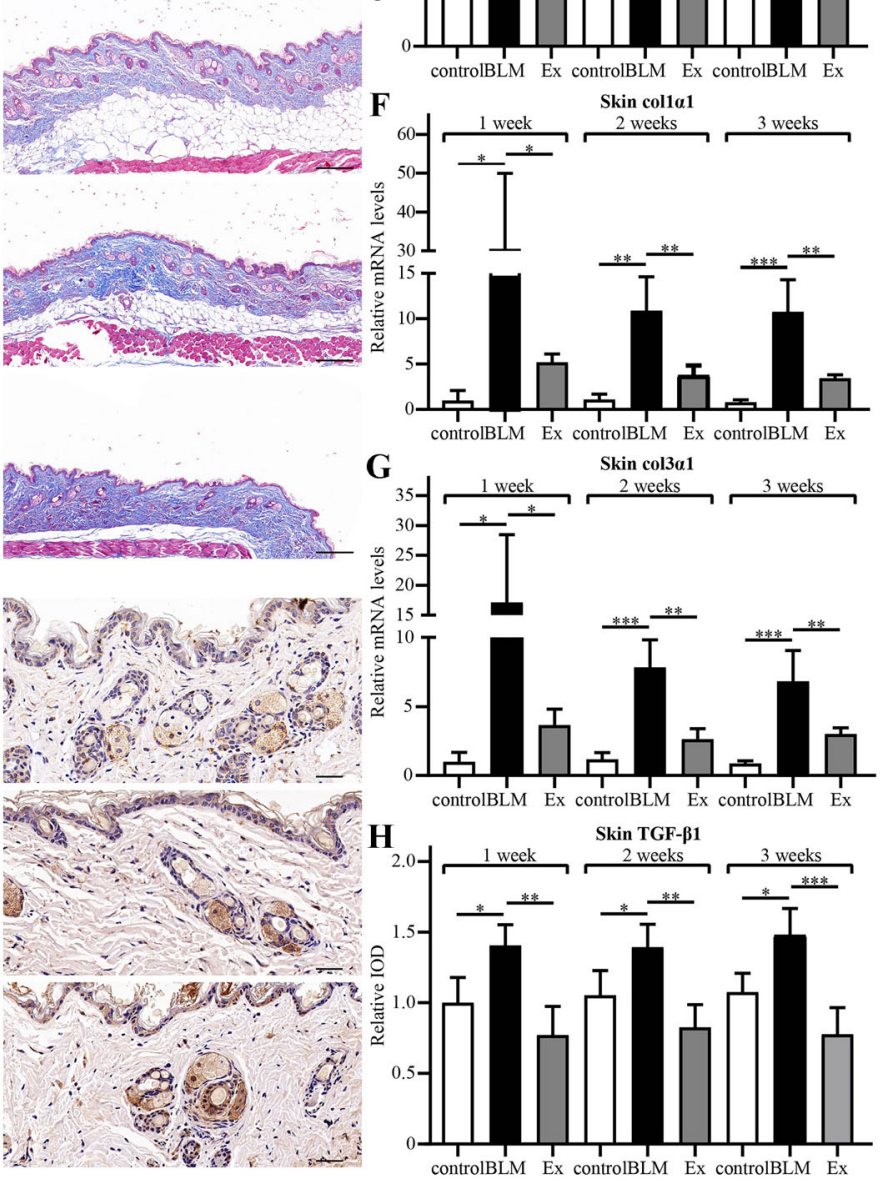

thickness and collagen content were quantified from $\mathrm{H} \& \mathrm{E}$ and Masson's staining respectively. F-G Col $1 \alpha 1$ and $\operatorname{col} 3 \alpha 1$ related to ECM were assessed by qPCR. H The protein expression level was quantified with IOD. Data are presented as the mean $\pm \mathrm{SD}$, $* p<0.05, * * p<0.01$, and $* * * p<0.005$

protein expression levels of IL-4 and IL-10 secreted by M2 macrophages were upregulated in the BLM group, but there was no statistically significant difference in IL-4 ( $p>0.05$ vs. the BLM group). HUMSCs-Ex reduced the 

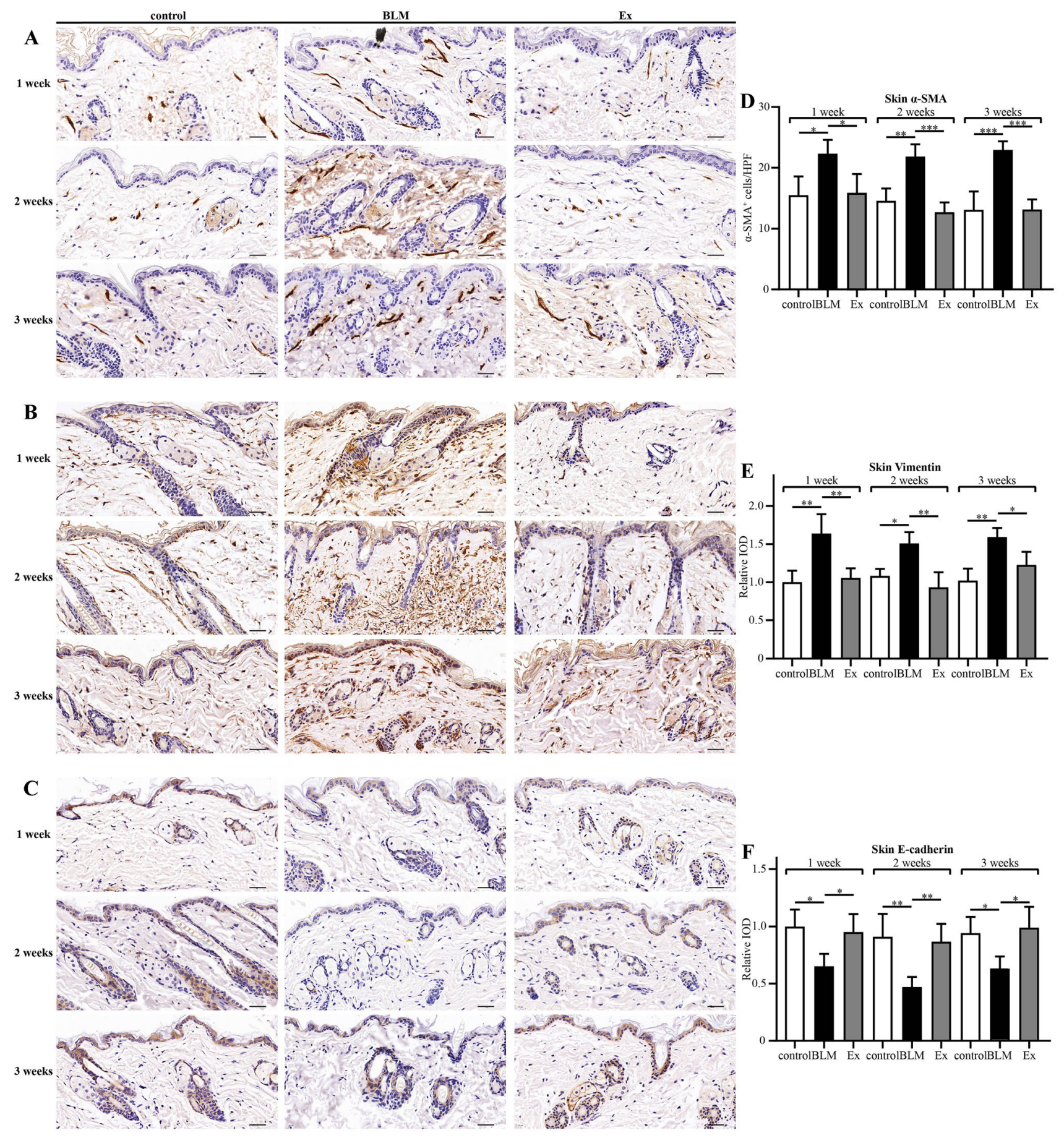

Fig. 3 HUMSCs-Ex restored EMT. A-C The distribution and expression of $\alpha$-SMA, vimentin and E-cadherin were assessed by immunohistochemical staining. Scale bars $=40 \mu \mathrm{m}$. D The number of $\alpha$-SMA ${ }^{+}$myofibroblasts was counted under each high power field.

protein expression levels of IL-4 and IL-10 (Fig. 4C, D). As shown in Fig. 4I, J, the result of Western blot results showed that the M1 macrophage polarization marker iNOS was upregulated and the M2 polarization macrophages marker Arg-1 was downregulated after HUMSCs-Ex

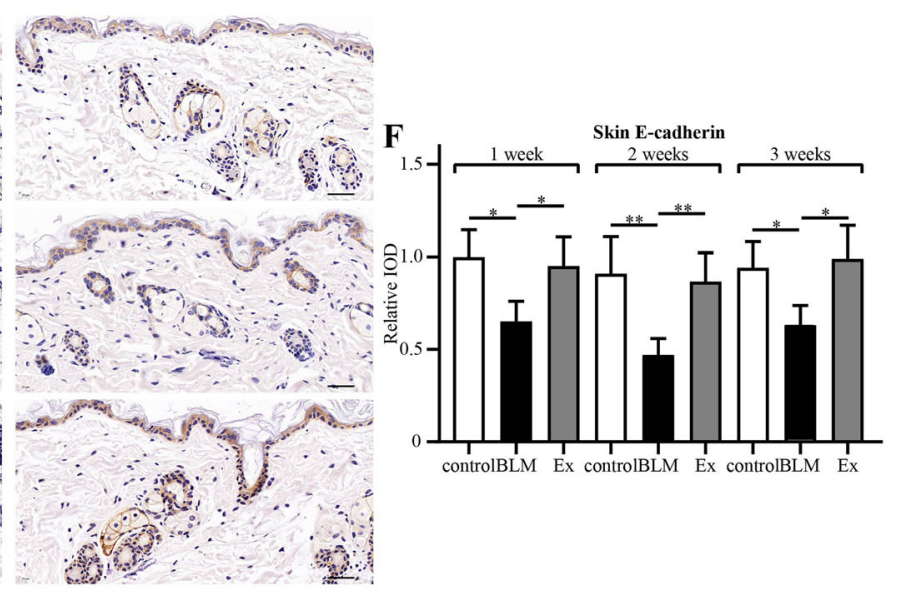

E-F The protein expression levels of vimentin and E-cadherin were quantified with IOD. Data are presented as the mean $\pm \mathrm{SD}$, $* p<0.05, * * p<0.01$, and $* * * p<0.005$

interference in a mouse model of scleroderma. To reflect the status of M1/M2 macrophages more clearly, we took the ratios of their related markers. M1 macrophages were depolarized, M2 macrophages were polarized in the BLM group, and HUMSCs-Ex repaired the destroyed balance 

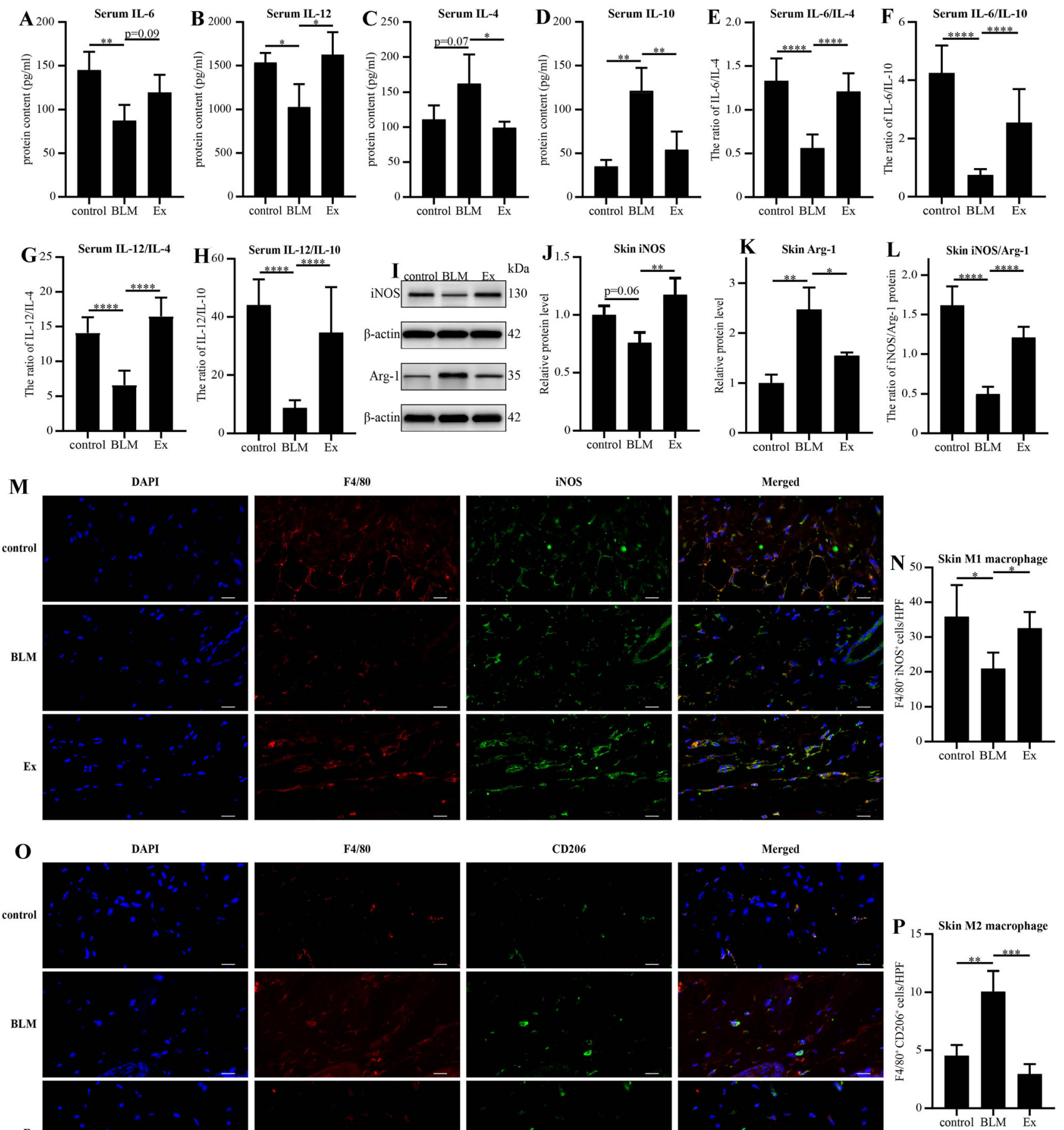

Fig. 4 HUMSCs-Ex modulated the balance of M1/M2 macrophages. A-D The ELISA results of the inflammatory cytokines IL-6, IL-12, IL-4 and IL-10 in serum. E-H The ratio of IL-6/IL-4, IL-6/IL-10, IL12/IL-4 and IL-12/IL-10 protein. I-K Western blot and quantification of iNOS and Arg-1 in skin. $\mathbf{L}$ The ratio of iNOS/Arg-1 protein. M-

(Fig. E-H and L) $(p<0.05$ the BLM group vs. the control group; $p<0.05$ the BLM group vs. the Ex group). Compared with the control group, the number of $\mathrm{F} 4 / 80^{+} \mathrm{iNOS}^{+}$
P Skin sections were stained with anti-F4/80 together with anti-iNOS or anti-CD206. Scale bars $=20 \mu \mathrm{m}$. The number of double-positive cells was counted under each high power field. Data are presented as the mean $\pm \mathrm{SD}, * p<0.05$

positive cells (M1 macrophages) decreased in the BLM group. HUMSCs-Ex treatment increased the number of M1 macrophages (Fig. 4M-N). Moreover, the number of F4/ 
$80^{+} \mathrm{CD}^{206^{+}}$positive cells (M2 macrophages) increased in the BLM group, and was attenuated by HUMSCs-Ex treatment (Fig. 4O-P).

\section{Discussion}

At present, combination treatment with methotrexate and corticosteroids is a conventional treatment for scleroderma, but it is associated with severe side effects such as pancytopenia [15]. Although biologic agents are playing an increasingly important role in the treatment of scleroderma, these therapies cannot reverse the progression of the disease [16]. Therefore, there is an urgent need to seek a safe and effective treatment for scleroderma. In this study, we established a mouse model of scleroderma as the object of study. As a rare disease, scleroderma is characterized by clinical heterogeneity, which is not conducive to experimental research. The bleomycin-induced mouse model of scleroderma can reflect the features of human scleroderma well, with simple establishment and high reproducibility $[17,18]$.

Although some studies have been conducted on MSCs for scleroderma, there are few reports of their use in the clinic. One of the existing studies shows that three months after a patient with refractory scleroderma injected with MSCs, the patient's ulcerations decreased and the blood circulation of their hands improved [19]; another study followed five patients for 44 months after receiving MSCs, with skin improvement occurring in three patients, and no adverse events occurring in five patients [20]. However, those studies lack large multicenter randomized controlled trials. While we cannot be completely sure of the therapeutic efficacy of MSCs, these studies suggest the possibility of new therapeutic approaches.

Studies have shown that bone marrow mesenchymal stem cells or adipose mesenchymal stem cells [21] or their exosomes [22] achieve good therapeutic effects in the treatment of sclerodermatous mice, but there is no report on HUMSCs-Ex. Almost all cells can release exosomes, but umbilical cord mesenchymal stem cells are cost-effective, productive and acceptable to gain [23]. As a group of MSCs derived from neonatal umbilical cord, umbilical cord mesenchymal stem cells have a stronger ability to differentiate [24]. Therefore, we used HUMSCs-Ex as a tool to investigate the possibility of treating scleroderma.

We observed that HUMSCs-Ex alleviated bleomycininduced skin fibrosis for at least 3 weeks. Additionally, we identified the key proteins $\alpha$-SMA, Vimentin, and E-cadherin, and found that HUMSCs-Ex inhibited the transition from the endothelial form to the mesenchymal form. Takahashi [25] analyzed the skin lesions of 11 patients with scleroderma and found that $\alpha$-SMA expression increased and E-cadherin expression decreased. Although HUMSCs-Ex has been described in some literature as having a similar ability to influence EMT [26, 27], this is the first time that HUMSCs-Ex have been discovered to regulate EMT in skin fibrosis. Organ fibrosis is the most important pathophysiological process of scleroderma, but vascular damage and immune disorders precede fibrosis [28]. Some scholars even believe that vascular injury can represent the pathogenesis of scleroderma, which is the basis of fibrosis [29]. A variety of cells, such as vascular endothelial cells and vascular smooth muscle cells, can be transformed into myofibroblasts through the EMT pathway, and the activated myofibroblasts can then synthesize collagen to result in ECM [30], which may express the interaction between proliferative vasculopathy and cutaneous fibrosis and their effect on the occurrence of scleroderma.

Activated macrophages can trigger a cascade of immune responses, and the loss of the balance of M1/M2 macrophages can lead to the occurrence of diseases. The most typical examples are M1 macrophages, which can cause acute inflammation and tissue damage, and M2 macrophages, which are involved in wound healing and induce fibrosis [31]. Skin CD204 and CD163, both phenotype markers of M2 macrophages, were significantly higher in scleroderma patients than in healthy subjects $[32,33]$. The elevation of serum CD163 in scleroderma patients was closely related to disease severity [34]. Several drugs have been found to treat scleroderma mice by inhibiting M2 macrophage polarization, such as nintedanib [34], glycyrrhizin [35], tamibarotene [36], anti-CX3 CL1 mAb [37] and WKYMVM [38]. We found that HUMSCs-Ex elevated M1 macrophage markers (IL-6, IL-12p40, iNOS) and inhibited M2 macrophage markers (IL-4, IL-10, Arg-1 and CD206) in a mouse model of scleroderma, thus suggesting that HUMSCs-Ex might play an antifibrotic role by regulating the M1/M2 macrophage balance.

In conclusion, HUMSCs-Ex stimulated macrophage phenotypes from M2 to M1 and suppressed EMT, which might explain why HUMSCs-Ex played an antifibrotic role in scleroderma. For the first time, our study provided preliminary evidence for the clinical treatment of HUMSCsEx in scleroderma.

\section{Declarations}

Conflict of interest The authors state no conflict of interest.

Ethical statement All animal experiments were complied with the ARRIVE guidelines and performed in accordance with the National Institutes of Health Guide for the Care and Use of Laboratory Animals. The experiments were approved by the Institutional Animal Care and Use Committee of Tongji University (No. TJ-HB-LAC2020-83). 
Supplementary InformationThe online version contains supplementary material available at https://doi.org/10.1007/s13770021-00405-5.

Open Access This article is licensed under a Creative Commons Attribution 4.0 International License, which permits use, sharing, adaptation, distribution and reproduction in any medium or format, as long as you give appropriate credit to the original author(s) and the source, provide a link to the Creative Commons licence, and indicate if changes were made. The images or other third party material in this article are included in the article's Creative Commons licence, unless indicated otherwise in a credit line to the material. If material is not included in the article's Creative Commons licence and your intended use is not permitted by statutory regulation or exceeds the permitted use, you will need to obtain permission directly from the copyright holder. To view a copy of this licence, visit http://creativecommons. org/licenses/by/4.0/.

\section{References}

1. Katsumoto TR, Whitfield ML, Connolly MK. The pathogenesis of systemic sclerosis. Annu Rev Pathol. 2011;6:509-37.

2. Chifflot H, Fautrel B, Sordet C, Chatelus E, Sibilia J. Incidence and prevalence of systemic sclerosis: a systematic literature review. Semin Arthritis Rheum. 2008;37:223-35.

3. Karassa FB, Ioannidis JP. Mortality in systemic sclerosis. Clin Exp Rheumatol. 2008;26:S85-93.

4. Fransen J, Popa-Diaconu D, Hesselstrand R, Carreira P, Valentini G, Beretta L, et al. Clinical prediction of 5-year survival in systemic sclerosis: validation of a simple prognostic model in EUSTAR centres. Ann Rheum Dis. 2011;70:1788-92.

5. Denton CP, Khanna D. Systemic sclerosis. Lancet. 2017;390:1685-99.

6. Christmann RB, Lafyatis R. The cytokine language of monocytes and macrophages in systemic sclerosis. Arthritis Res Ther. 2010;12:146.

7. Zhang Z, Wu Y, Wu B, Qi Q, Li H, Lu H, et al. DZ2002 ameliorates fibrosis, inflammation, and vasculopathy in experimental systemic sclerosis models. Arthritis Res Ther. 2019;21:290.

8. Thiery JP, Acloque H, Huang RY, Nieto MA. Epithelial-mesenchymal transitions in development and disease. Cell. 2009;139:871-90.

9. Scita G, Di Fiore PP. The endocytic matrix. Nature. 2010;463:464-73.

10. Rostom DM, Attia N, Khalifa HM, Abou Nazel MW, El Sabaawy EA. The therapeutic potential of extracellular vesicles versus mesenchymal stem cells in liver damage. Tissue Eng Regen Med. 2020;17:537-52.

11. Ding M, Shen Y, Wang P, Xie Z, Xu S, Zhu Z, et al. Exosomes isolated from human umbilical cord mesenchymal stem cells alleviate neuroinflammation and reduce amyloid-beta deposition by modulating microglial activation in alzheimer's disease. Neurochem Res. 2018;43:2165-77.

12. Li T, Yan Y, Wang B, Qian H, Zhang X, Shen L, et al. Exosomes derived from human umbilical cord mesenchymal stem cells alleviate liver fibrosis. Stem Cells Dev. 2013;22:845-54.

13. Luo Z, Lin J, Sun Y, Wang C, Chen J. Bone marrow stromal cellderived exosomes promote muscle healing following contusion through macrophage polarization. Stem Cells Dev. 2021;30:135-48.

14. Su TI, Khanna D, Furst DE, Danovitch G, Burger C, Maranian P, et al. Rapamycin versus methotrexate in early diffuse systemic sclerosis: results from a randomized, single-blind pilot study. Arthritis Rheum. 2009;60:3821-30.
15. Denton CP, Engelhart M, Tvede N, Wilson H, Khan K, Shiwen $\mathrm{X}$, et al. An open-label pilot study of infliximab therapy in diffuse cutaneous systemic sclerosis. Ann Rheum Dis. 2009;68:1433-9.

16. Yamamoto T, Takagawa S, Katayama I, Yamazaki K, Hamazaki Y, Shinkai H, et al. Animal model of sclerotic skin. I: Local injections of bleomycin induce sclerotic skin mimicking scleroderma. J Invest Dermatol. 1999;112:456-62.

17. Marangoni RG, Varga J, Tourtellotte WG. Animal models of scleroderma: recent progress. Curr Opin Rheumatol. 2016;28:561-70

18. Christopeit M, Schendel M, Föll J, Müller LP, Keysser G, Behre G. Marked improvement of severe progressive systemic sclerosis after transplantation of mesenchymal stem cells from an allogeneic haploidentical-related donor mediated by ligation of CD137L. Leukemia. 2008;22:1062-4.

19. Keyszer G, Christopeit M, Fick S, Schendel M, Taute BM, Behre $\mathrm{G}$, et al. Treatment of severe progressive systemic sclerosis with transplantation of mesenchymal stromal cells from allogeneic related donors: report of five cases. Arthritis Rheum. 2011;63:2540-2.

20. Okamura A, Matsushita T, Komuro A, Kobayashi T, Maeda S, Hamaguchi $\mathrm{Y}$, et al. Adipose-derived stromal/stem cells successfully attenuate the fibrosis of scleroderma mouse models. Int J Rheum Dis. 2020;23:216-25.

21. Jin J, Ou Q, Wang Z, Tian H, Xu JY, Gao F, et al. BMSC-derived extracellular vesicles intervened the pathogenic changes of scleroderma in mice through miRNAs. Stem Cell Res Ther. 2021;12:327.

22. Fang S, Xu C, Zhang Y, Xue C, Yang C, Bi H, et al. Umbilical cord-derived mesenchymal stem cell-derived exosomal microRNAs suppress myofibroblast differentiation by inhibiting the transforming growth factor- $\beta /$ SMAD2 pathway during wound healing. Stem Cells Transl Med. 2016;5:1425-39.

23. Wang M, Yuan Q, Xie L. Mesenchymal stem cell-based immunomodulation: properties and clinical application. Stem Cells Int. 2018;2018:3057624.

24. Takahashi M, Akamatsu H, Yagami A, Hasegawa S, Ohgo S, Abe $\mathrm{M}$, et al. Epithelial-mesenchymal transition of the eccrine glands is involved in skin fibrosis in morphea. J Dermatol. 2013;40:720-5.

25. Xiao K, He W, Guan W, Hou F, Yan P, Xu J, et al. Mesenchymal stem cells reverse EMT process through blocking the activation of NF- $\kappa \mathrm{B}$ and Hedgehog pathways in LPS-induced acute lung injury. Cell Death Dis. 2020;11:863.

26. Yao Y, Chen R, Wang G, Zhang Y, Liu F. Exosomes derived from mesenchymal stem cells reverse EMT via TGF- $\beta 1 / \mathrm{Smad}$ pathway and promote repair of damaged endometrium. Stem Cell Res Ther. 2019;10:225.

27. Gabrielli A, Avvedimento EV, Krieg T. Scleroderma. N Engl J Med. 2009;360:1989-2003.

28. Matucci-Cerinic M, Kahaleh B, Wigley FM. Review: evidence that systemic sclerosis is a vascular disease. Arthritis Rheum. 2013;65:1953-62.

29. Zvaifler NJ. Relevance of the stroma and epithelial-mesenchymal transition (EMT) for the rheumatic diseases. Arthritis Res Ther. 2006;8:210.

30. Mosser DM, Edwards JP. Exploring the full spectrum of macrophage activation. Nat Rev Immunol. 2008;8:958-69.

31. Higashi-Kuwata $\mathrm{N}$, Makino $\mathrm{T}$, Inoue $\mathrm{Y}$, Takeya $\mathrm{M}$, Ihn $\mathrm{H}$. Alternatively activated macrophages (M2 macrophages) in the skin of patient with localized scleroderma. Exp Dermatol. 2009;18:727-9.

32. Higashi-Kuwata N, Jinnin M, Makino T, Fukushima S, Inoue $\mathrm{Y}$, Muchemwa FC, et al. Characterization of monocyte/macrophage subsets in the skin and peripheral blood derived from patients with systemic sclerosis. Arthritis Res Ther. 2010;12:R128. 
33. Bielecki M, Kowal K, Lapinska A, Chyczewski L, Kowal-Bielecka O. Increased release of soluble CD163 by the peripheral blood mononuclear cells is associated with worse prognosis in patients with systemic sclerosis. Adv Med Sci. 2013;58:126-33.

34. Huang J, Maier C, Zhang Y, Soare A, Dees C, Beyer C, et al. Nintedanib inhibits macrophage activation and ameliorates vascular and fibrotic manifestations in the Fra2 mouse model of systemic sclerosis. Ann Rheum Dis. 2017;76:1941-8.

35. Yamashita T, Asano Y, Taniguchi T, Nakamura K, Saigusa R, Miura S, et al. Glycyrrhizin ameliorates fibrosis, vasculopathy, and inflammation in animal models of systemic sclerosis. J Invest Dermatol. 2017;137:631-40.

36. Toyama T, Asano Y, Akamata K, Noda S, Taniguchi T, Takahashi $\mathrm{T}$, et al. Tamibarotene ameliorates bleomycin-induced dermal fibrosis by modulating phenotypes of fibroblasts, endothelial cells, and immune cells. J Invest Dermatol. 2016;136:387-98.

37. Luong VH, Utsunomiya A, Chino T, Doanh LH, Matsushita T, Obara $\mathrm{T}$, et al. Inhibition of the progression of skin inflammation, fibrosis, and vascular injury by blockade of the $\mathrm{CX}_{3} \mathrm{CL} 1 /$ $\mathrm{CX}_{3} \mathrm{CR} 1$ pathway in experimental mouse models of systemic sclerosis. Arthritis Rheumatol. 2019;71:1923-34.

38. Park GT, Kwon YW, Lee TW, Kwon SG, Ko HC, Kim MB, et al. Formyl peptide receptor 2 activation ameliorates dermal fibrosis and inflammation in bleomycin-induced scleroderma. Front Immunol. 2019;10:2095.

Publisher's Note Springer Nature remains neutral with regard to jurisdictional claims in published maps and institutional affiliations. 